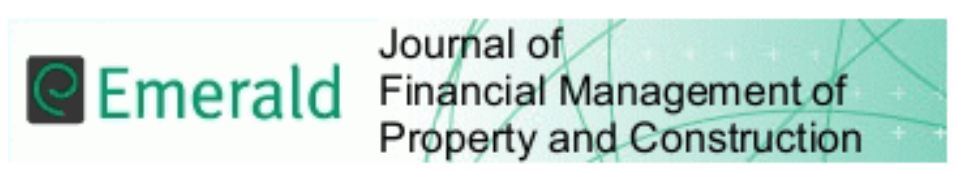

\title{
Prioritisation of Resilience Criteria and Performance Indicators for Road Emergencies Crisis Response: An Analytic Hierarchy Process (AHP) Approach
}

\begin{tabular}{|r|l|}
\hline Journal: & Journal of Financial Management of Property and Construction \\
\hline Manuscript ID & JFMPC-11-2021-0065 \\
\hline Manuscript Type: & Research Paper \\
\hline Keywords: & $\begin{array}{l}\text { Resilience Criteria, Performance Indicators, Road Emergencies Crisis } \\
\text { Response, Analytic Hierarchy Process, Delphi Method }\end{array}$ \\
\hline \multicolumn{2}{|l}{}
\end{tabular}

\section{SCHOLARONE \\ Manuscripts}




\section{Prioritisation of Resilience Criteria and Performance Indicators for Road Emergencies Crisis Response: An Analytic Hierarchy Process (AHP) Approach}

\section{Introduction}

Contemporary society is increasingly exposed to high levels of unpredictability, volatility and a multitude of risks that can result in significant human, physical, economic or environmental loss. Historical occurrences have consistently underlined the need for strong resilience and readiness capabilities to ensure the ability to effectively respond, alleviate and contain the impacts of crises or disaster events (Boin et al., 2016). Changes in the global ecological environment, human behaviour and rapid urbanisation have amplified and accelerated the gravity of events (Dranseika and Gordijn, 2018; Borraz and Cabane, 2017).

While many studies and models have addressed disaster management (Lettieri et al., 2009; Perry, 2007; Coppola, 2006), research into road emergency crisis readiness is scarce and less established. The economic consequences of the road traffic crisis are very significant, in terms of both lost productivity and all healthcare resources needed. This includes injuries and fatalities, property damage, workplace and household productivity losses, medical costs, traffic congestion and other costs. However, there is a gap in published research for a comprehensive crisis readiness and response framework that defines the critical success factors and performance indicators at a local or agency level. Thus, there is a need for the development of specific resilience indicators that will support the development and evaluation of crisis readiness.

This study is influenced by the problem context in the UAE, which has experienced an increase in road traffic crises in recent years triggered by increasing traffic and natural meteorological events such as fog and sand storms. As traffic on the UAE's roads has steadily increased, there has been a concomitant rise in road traffic accidents. Road fatalities are the second largest cause of death in the UAE after heart disease (DoT, 2018). Fatality rates are three times compared to those in the UK, at 7.95 deaths per 100,000 inhabitants in 2016, a rise of 7.4\% over 2015 (RoadSafetyUAE, 2018). Data from the World Health Organisation (2015) put this figure much higher at 18.1 traffic-related fatalities per 100,000 inhabitants. The UAE lags behind Western economies such as the UK, Germany and France, in terms of effectiveness of its road crisis response efforts.

The significance of crisis readiness and response is underscored by the increase and impact of road traffic globally. The costs to economies of road traffic incidents has risen to nearly $3 \%$ of annual gross domestic product (GDP), while over $90 \%$ of road traffic deaths occur in low and middleincome countries (WHO, 2020). The rapid development and urbanisation of developing countries such as the UAE lacking a mature regulatory framework, can have negative consequences for public health and economic productivity. For instance, response time for road traffic crises and emergencies for the UAE are significantly higher than benchmarks for global best practice. Average response times among major global cities in 2017 was 7:06 minutes for New York, 12 minutes for Singapore, and 14:44 minutes for London. In comparison, internal data from the Ministry of Interior shows that the response time in the UAE was 11.97 seconds, which represents a significant improvement over the 2016 response time of 13.16 (Mol, 2017). Despite gradual reductions in average response times over the last three years, there is a wide gap in the UAE between current practice and the national target of 4 minutes. A key strategy in optimising response times is focused on improving crisis readiness.

The issue of response is situated within broader internal and external factors that influence the 
capacity for authorities to respond to traffic crises. In the UAE, recent responses to crises revealed that effective coordination is hampered by an absence of appropriate policies and mechanisms and understanding of how coordination can be optimised (Alteneiji, 2015). In particular enhancing coordination between federal and local levels during the management of disasters has been cited as a priority (Al-Marzooqi et al., 2017; Almarzouqi, 2017; Alteneiji, 2015). While NCEMA is the federal level authority charged with crisis management during disasters, some overlap has been noted in relation to how prevention, preparedness and recovery efforts are coordinated with the police force, which clearly assume a central role in leading responses and managing crises and disasters. Duplication of roles, communication gaps, and speed of response are identified as key challenges (Al-Marzooqi et al., 2017).

Broader evaluation of the crisis response components and processes has been recognised as critical to enhancing the responsiveness of police to road traffic crises. This paper addresses a lack of research and understanding of the key dimensions and components of crisis readiness for road traffic incidents. Whereas crisis management and disaster resilience are recognised as major mechanisms to minimise the adverse impacts (Cutter et al., 2010), there is significant opportunity to develop specific indicators to support the advancement and evaluation of crisis readiness. Thus the central goal of this study is focused on enhancing the organisational effectiveness of law enforcement agencies within the United Arab Emirates (UAE) to effectively respond to crisis situations and disasters. This paper addresses this issue by conceptualising and validating a comprehensive strategic framework for crisis readiness that contributes a model to support readiness and response planning to improve police response times to road traffic crises.

\section{Literature Review}

While the notion of crisis readiness extends over a range of disciplines (WTTC, 2019) there is a lack of a universally accepted definition (Ritchie et al.,
2011). It has been used interchangeably with preparedness and represents a key focus of this research. It has increasingly been adopted in the crisis management literature to reflect a specific state or phase in the process. In applying the concept of crisis readiness to this research context, the optimisation of traffic agencies' response times can be evaluated from the perspective of individual and organisational state of mind "as a planned process of resource allocation and deployment" (Rousaki and Alcott 2006, p.575).

An early definition by Reilly $(1987, p .80)$ describes crisis readiness as "the readiness to cope with the uncertainty and change engendered by a crisis". Rousaki and Alcott (2006) define it as both a planned process of resource allocation and deployment, and also readiness in state of mind both at individual and organisational levels.

The characteristics of crisis readiness have been identified as a conscious and proactive orientation towards preparing for the inevitable occurrence of crises (Light and Morgan, 2008; Sheaffer and ManoNegrin, 2003). Light and Morgan (2008) emphasise that the organisational ability to effectively respond to and recover from major external events reflects the desired outcome of crisis management, organisational preparedness, business continuity planning and other organisational processes. According to Shrivastava and Mitroff (1987) the prevention and management of crises is evidently possible but only when an in-depth understanding exists of the risks and nature of the crises. This understanding in turn provides the basis for the initiation and development of strategic plans and programmes. In an early conceptualisation of a crisis readiness construct, Reilly (1987) identified six key components: organisational capacity for rapid response to crises; both managerial awareness of and access to crisis management plans and resources; adequacy of organisational strategic crisis planning; ability to manage media during the crisis, and perceived probability of a crisis occurring to or within an organisation. 


\section{Conceptualising Crisis Readiness}

As yet no comprehensive framework has been developed or validated in respect of crisis readiness dimensions and elements generally or in the specific context of road traffic crisis management. Thus the theoretical elements of crisis readiness are identified from an analysis of these theories and frameworks and models. In mapping the key dimensions and elements of crisis readiness fourteen distinct dimensions of crisis readiness were identified. A systematic search was conducted of social science journal databases guided by relevant keywords for the searches. The review integrated the key national models and institutional frameworks for disaster and crisis preparedness: Strategic Framework for Emergency Preparedness (WHO, 2017); Common Framework for Preparedness (CFP) by the InterAgency Standing Committee (IASC, 2013); The FEMA Capability Assessment for Readiness (CAR) framework (FEMA, 2001), and the national disaster emergency preparedness framework by Sutton and Tierney (2006). The databases searched were ProQuest, EBSCO, PsychINFO and Google Scholar. Further searches used Google to identify grey literature such as reports published by international bodies or government organisations. Initial screening was based on titles and abstract to shortlist studies and literature that addressed emergency, disaster or crisis themes focused on response, readiness and preparedness. Sources were then reviewed on the grounds of relevance to the research question, theoretical and methodological rigour (Anderson et al. 2001) and application in the practice. A total of 58 papers formed the basis of the conceptual framework proposed.

The literature points to several overarching principles drawn from crisis readiness frameworks and models that underpin crisis readiness planning and preparation: whole-of-society approach, joint planning and coordination, political leadership and commitment, a comprehensive multi-hazard approach and a continuous process of learning and capacitybuilding (WHO, 2017; IASC, 2013; Sutton and Tierney, 2006).
In the USA the Capability Assessment for Readiness (CAR) (FEMA, 2001) is a survey instrument developed by the US Federal Emergency Management Agency (FEMA) to assess state capabilities and operational readiness for mitigation, preparedness, response and recovery from emergencies and disasters. Thirteen emergency management functions are identified and measured using national level performance criteria. The model represents a closed, ongoing process consisting of five stages of planning, organising and equipping, training, exercising and evaluation which initiates the cycle again. A criticism of this however is the lack of integration of key elements of crisis readiness within the cycle including risk assessment, early warning, information and communication and public education. The UK crisis readiness cycle is viewed as one of the most holistic and detailed crisis readiness cycles (Alteneiji, 2015). It contrasts with the US model in including two major processes of consult and embed which function as guidelines for the readiness phase (CCA, 2004). The consult component addresses risk assessment and the embed component implementation processes: communication, governance, training and evaluation (CCA, 2004). The Australian framework is designed to address the significant variety of natural and non-natural hazards that the country faces (EMA, 2004). The model addresses the entire timeline of the crisis cycle that decomposes elements of readiness into specific activities which can be easily understood and implemented.

In addition to national models, a review of the literature identifies key crisis readiness frameworks, models and instruments that provide insights into principles and core components. Four of the prominent frameworks originate from international or national institutions and agencies responsible for crisis or emergency response, including FEMA's CAR model. The Common Framework for Preparedness is a disaster risk management framework developed by the InterAgency Standing Committee and the United Nations (IASC, 2013). This enables international and regional actors to cooperate with governments and national institutions. A humanitarian approach is emphasised based on 
human rights norms and which underpin the operationalisation of the framework. The model provides a systematic approach based on eight key components to collectively assess capabilities and needs and develop and implement programmes and plans to enhance preparedness.

A theoretical model advanced by Sutton and Tierney (2006) draws on a systematic review of diverse practitioner, government and academic sources on preparedness and planning including research instruments, preparedness guidance and checklists, guidance from federal agencies, best practices, and scholarly and business journals. This identified eight dimensions of preparedness for households, businesses and communities and organisations implemented as a continuous and proactive process that incorporates learning and broad engagement.

Similarly, broad engagement is a key theme in the Strategic Framework for Emergency Preparedness developed by the World Health Organisation (WHO). This is based on strong principles of political and community engagement and commitment. Key elements grouped around three main areas of governance, capacities and resources support the determination of priorities and the strengthening of operational capacities (WHO, 2017).

\section{Key Dimensions of Crisis Readiness}

The conceptualisation of crisis readiness draws on the convergence in theory and praxis on key principles and elements. The literature points to multiple distinct but interrelated factors. Fourteen elements have been identified which are integrated into a comprehensive conceptual framework that guides the development of a crisis readiness framework. These elements have been drawn from models and frameworks from different contexts and sectors.

Risk assessment is common to all the frameworks and focuses on identification and evaluation of hazards and risks, vulnerabilities, and capacities to determine priorities for emergency preparedness (WHO, 2017; IASC, 2013). There is consensus in the literature on the concept of community participation in the risk assessment process specifically in supporting multi-hazard, multisectoral and multi-level risk and capacity assessments (WHO, 2017; Ikeda, 2010; Alexander, 2009; Nagasaka, 2006; CCA, 2004).

\section{Early Warning}

In the crisis readiness literature risk assessment and early warning are integrated components as risk assessment and environmental monitoring in terms of forecasting and preparing for risks. Early warning can have a significant impact on crisis response times by enabling precipitous and timely activation of crisis plans, teams and resources. The features in most models address the preparedness of alert systems at a local, national, regional and international level (IASC, 2013).

\section{Legal and Institutional Frameworks}

Legal and institutional frameworks have some importance for crisis response times in terms of framing and underpinning mechanisms, structures and processes which activate, coordinate, and scale up crisis response at different levels from local to national and beyond (WHO, 1999). In the majority of frameworks, the legal and regulatory aspect is addressed in terms of the development and maintenance of emergency management programmes (WHO, 2017; IASC, 2013; FEMA, 2001). Recurring regulatory themes include crisis readiness legislation; integrated emergency preparedness; national plans and disaster authorities; cross-sectoral and sectoral frameworks; resource allocation, funding mechanisms, and regional and international agreements (WHO, 2017; IASC, 2013).

\section{Resources}

Resources can have a major and direct influence on the ability of road traffic agencies to respond appropriately to mitigate the worst impacts of a crisis on human life and property (EU, 2018). This component is identified in all frameworks and is concerned with appropriate resource allocation to ensure coping capacity and to enable the availability and accessibility of funding for crisis response (WHO, 2017; IASC, 2013; Sutton and Tierney, 2006; FEMA, 2001). Much of the discussion focused predominantly on the 
availability and accessibility of financial, technical, and human resources. This dimension is identified as a key antecedent both in terms of building a state of crisis readiness and to support allocation and deployment of response teams, technical equipment, and other resources (WHO, 2017; Zemp, 2010; Light and Morgan, 2008; Hoffer Gittel et al., 2006; Kovoor-Misra, 1995).

\section{Coordination}

Coordination is a recurrent theme in the crisis readiness literature across all the models. Rapid response requires effective communication patterns and the coordination of resources, equipment, and skills to enable seamless collaborative action (Abbasi et al., 2018). This is characterised in terms of government coordination mechanisms, leadership structures and inter-agency coordination at all levels and across sectors (WHO, 2017; IASC, 2013).

\section{Information Management and Communication}

Central to most models is the development of information management systems and communication systems at sector, national, regional and international levels for rapid dissemination of crisis communication in a timely response that minimises the impacts of the crisis. (Collins et al., 2016; IASC, 2013; FEMA, 2001). The literature converges around five key elements: communication systems; ICT technologies; information gathering and dissemination: data analysis and simulation tools; and communication response.

\section{Response Planning}

Response planning is represented in all the crisis preparedness models reviewed, associated with community preparedness, preparedness programmes and contingency planning (IASC, 2013), risk assessment and optimisation of crisis response times (OECD, 2013). Key principles emphasise engagement of actors in the planning process and definition of key roles and responsibilities (OECD, 2013). Models emphasise learning and evaluation processes that incorporate continual learning and improvement and feedback mechanisms (Kartez and Lindell, 1987).

\section{Training}

Training is recognised as one of the most pivotal factors in crisis readiness that can significantly contribute to lowering crisis response times (FEMA, 2015). Comprehensive assessment, design and planning is established as the foundation and initial basis for developing capabilities of all personnel at all levels internally and externally including multi-stakeholders such as emergency volunteers (WHO, 2017; FEMA, 2015; Henstra, 2010; Dillon et al., 2009). Key training elements address all-hazards planning, role-specific knowledge and skills, national context training opportunities, next generation core competencies (Feldmann-Jensen et al., 2017; Eriksson, 2015; IASC, 2013; EMAP, 2004; FEMA, 2001). Effective crisis leadership in the response phase of crises has been associated with a range of specific competencies and practices. Competency needs are identified based on the demands that crisis places on leadership for strong calm leaders, communication, delegation, pragmatism and responsiveness (Cronin, 2015; Van Wart and Kapucu, 2011)

\section{Exercises}

Exercises are recognised as a distinct dimension of training and preparedness (IASC, 2013; FEMA, 2001) to develop familiarity and conditioning to crisis situations (OECD, 2013; Dillon et al. 2009; FEMA, 2001; Drennan et al., 2014). Existing models stipulate regularly scheduled simulations, drills and practical exercises for local, national and/or international actors (IASC, 2013; Sutton and Tierney, 2006; FEMA, 2001). Exercises are associated with a number of positive outcomes that enhance crisis readiness: enhancing cognitive sense-making abilities; revealing weaknesses, and providing data (Cronin, 2015; Lampel et al., 2009; Gordon, 2002) in crisis situations amongst all levels of staff (Cronin, 2015; OECD, 2013; Acosta et al., 2009).

\section{Logistics and Facilities}

Logistics and facilities planning are reflected in all models and are addressed in relation to 
contingency and standby arrangements for crises including stockpiling of resources and contingency partnership resource and supply agreements (IASC, 2013). Most frameworks emphasise the development of logistics capabilities and mechanisms which fall into four key categories of material management, property management, facility management and transportation management (WHO, 2017; Sutton and Tierney, 2006; FEMA, 2001).

\section{Public Education}

Public education definitions emphasise a comprehensive process in which the public is informed and educated on crisis risks (Alexander, 2012). Public education is considered in the majority of models that emphasise an effective public education programme on local or national hazards and risks to enhance community crisis preparedness (WHO, 2017; Sutton and Tierney, 2006; FEMA, 2001).

\section{Hazard Management}

Hazards represent a unit of analysis and point of focus towards which all effort and resources for crisis readiness and response are directed. Hazards are defined as events, situations, processes or substances which are actual or potential sources of harm (NRC, 2006). The management of hazards is a component of crisis readiness in two of the models reviewed advocating a systematic approach to identification, assessment and mitigation of hazards posing significant threats (Sutton and Tierney, 2006; FEMA, 2001). The use of common crisis readiness functions that operate across all hazards boundaries is a key feature and includes functionalities such as: direction and control, warning and communication, continuity of government and operations, maintenance of essential public services, and resource management. The identification and assessment of hazards links to the risk assessment process in crisis readiness in much of the literature (WHO, 2017; IASC, 2013). Nevertheless, Sutton and Tierney (2006) identify specific core features designed to lead to future mitigative actions including the conduct of hazard, impact and vulnerability assessments and detailed understanding of the impacts on populations and facilities, structures, and infrastructure.

\section{Operations and Procedures}

Coordination and integration among different crisis responders is a key function that influences crisis response times and determines the speed with which agencies can activate core capabilities efficiently across all key stakeholders. The FEMA framework uniquely identifies operations and procedures as a standalone component within the crisis readiness framework (FEMA, 2001). It defines this as the development, coordination and implementation of operational policies, plans and procedures for crisis management. Effectiveness in this area is viewed as fundamental for regional crisis management structures to prepare, respond and recover from crises. The aim is to create and maintain a coordinated and integrated operational structure and process that effectively unifies all key stakeholders and enables the activation of core capabilities.

\section{Recovery Initiation}

The concept of recovery initiation was identified as a key component in half of the models (WHO, 2017; Sutton and Tierney, 2006). Restoration of critical services and facilities such as utilities and transport are considered the basis for early recovery activities and containing further impacts (WHO, 2017; Sutton and Tierney, 2006).

\section{Property Protection}

Property protection is identified by Sutton and Tierney (2006) in their systematic review as a further component. In terms of road traffic crisis responses, this might reflect the speed and effectiveness of agencies for expedient action to prevent loss or damage to property, facilities, buildings, equipment, to secure critical records and ensure the maintenance of critical functions during crises (Sutton and Tierney, 2006).

\section{Methodology}

A single case study strategy was adopted based on a mixed method study to conceptualise and verify a framework for crisis readiness. The Delphi Method was employed as the primary data collection mechanism to gather qualitative and 
quantitative data. Questionnaire methods and the Analytical Hierarchy Process (AHP) are integrated into the Delphi method to complete the research design. Online questionnaires will be the main methods used to collect different types of data at each stage to address the research goals.

The Analytical Hierarchical Process is a comprehensive framework that allows decisionmakers to generate multi-objective, multi-factor and multicriteria decisions on any number of alternatives (Willyard and McClees, 1987). The approach allows the incorporation of objective and quantitative aspects as well as qualitative facets of complex problems to be reconstructed into a coherent decision-making model. Complex problems are broken down into defined hierarchies of categories and elements which are ranked by pairwise comparison to establish the priorities or preferences within each hierarchy. This provides a weighting for each category and element within a category as well as a consistency ratio which facilitates assessment of the consistency of the data (Saaty, 1978). The process can be applied to different complex problems with a variety of decision analyses, allowing decisionmakers to identify and determine ratio scale priorities rather than assigning them arbitrarily (Richey and Grinnell, 2004).

Table 1 Structure of Delphi Process

\begin{tabular}{llll}
\hline & Method & Type & Analysis \\
\hline Round 1 & Open-Ended & Qualitative & $\begin{array}{l}\text { Thematic } \\
\text { Questionnaire }\end{array}$ \\
& & Analysis \\
\hline Round 2 & Semi-structured & Qualitative- & Thematic \\
& Questionnaire & Quantitative & Analysis \\
& & & - \\
& & & Descriptive \\
& & & Analysis \\
\hline Round 3 & Pairwise & Quantitative & AHP \\
& Comparison & & Analysis \\
\hline Round 4 & Open-Ended & Qualitative & Thematic \\
& Questionnaire & & Analysis \\
\hline
\end{tabular}

In the first round of the Delphi process as indicated in Table 1 members completed an unstructured online questionnaire to collect qualitative data about what factors and key performance indicators for each of the fourteen crisis readiness criteria were important for improving road traffic response times. In round two each Delphi participant was emailed a semi-structured questionnaire which integrated all the factors and measures generated from the first round. Participants reviewed and rated each criterion as well as their associated factors and performance measures using a Likert rating scale to denote perceived importance. In round three participants completed an Analytical Hierarchy Process form to derive the criteria, sub-criteria and key performance indicators evaluated as most important for optimising road traffic response times. The results from this round were then analysed and fed back to participants in the fourth and final round of the Delphi in which a final questionnaire was presented. This contained open-ended questions on the proposed strategic framework for improving road traffic crisis response times. This provided a final opportunity to participants to revise and refine their judgements and finalise the strategic framework based on the research findings.

A total of 16 practitioners participated in the study sampled from UAE police forces, NCEMA, Civil Defence directorates, government and federal and local road transport authorities as shown in Table 2.

Table 2 Participant Sample

\begin{tabular}{|c|c|}
\hline Sector & Organisation \\
\hline \multirow{3}{*}{ Law Enforcement } & $\begin{array}{l}\text { Abu Dhabi Police Traffic and } \\
\text { Patrols Directorate }\end{array}$ \\
\hline & $\begin{array}{l}\text { Dubai Police General Department } \\
\text { of Traffic }\end{array}$ \\
\hline & $\begin{array}{l}\text { Dubai Police Department of } \\
\text { Transport and Rescue }\end{array}$ \\
\hline \multirow{4}{*}{ Civil Defence } & Civil Defence General Command \\
\hline & Civil Defence Fire and Rescue \\
\hline & Dubai Civil Defence Directorate \\
\hline & $\begin{array}{l}\text { Abu Dhabi Civil Defence } \\
\text { Directorate }\end{array}$ \\
\hline \multirow{6}{*}{$\begin{array}{c}\text { Emergency and } \\
\text { Crisis } \\
\text { Management }\end{array}$} & $\begin{array}{l}\text { NCEMA Planning and } \\
\text { Preparedness Department }\end{array}$ \\
\hline & NCEMA Operation Department \\
\hline & NCEMA Local Centers Department \\
\hline & NCEMA Information \& \\
\hline & $\begin{array}{l}\text { Communication Technology } \\
\text { Department }\end{array}$ \\
\hline & $\begin{array}{l}\text { NCEMA Safety and Prevention } \\
\text { Department }\end{array}$ \\
\hline \multirow[b]{2}{*}{ Government } & Ministry of Interior \\
\hline & $\begin{array}{l}\text { Federal Transport Authority - Land } \\
\text { and Maritime }\end{array}$ \\
\hline
\end{tabular}


Abu Dhabi Department of

Transport

Dubai Road Traffic Authority

In this study the data was collected from participants from a cross-section of public sector directorates and command authorities at federal and local level that have key roles and responsibilities for road traffic crisis readiness and response. The selected participants possessed at least ten years of experience within emergency and crisis response and additionally had operational and strategic knowledge of crisis response practices and were involved in strategic and operational decision-making processes.

\section{AHP Process}

The quantitative data from the AHP was analysed in a systematic manner in accordance with established procedures. These comprised four main steps: definition of hierarchical framework; calculation of pairwise comparisons; synthesis and calculation of judgements for prioritisation of weights; and evaluation of the consistency of the results (Al-Shehri et al., 2015; Cabala, 2010).

In the first step a hierarchical framework was defined that consisted of ten criteria for crisis readiness based on the literature review that pointed to the existence of fourteen key dimensions of crisis readiness. In earlier Delphi phases participants provided qualitative feedback on the key relative importance of these fourteen criteria. A final ten criteria were shortlisted and formed the basis of the hierarchical framework for the AHP: Risk and Hazard Management; Legal and Institutional Frameworks; Resources; Coordination; Information Management and Communication; Response Planning; Early Warning; Training; Recovery Initiation, and Property Protection.

Pairwise comparisons were then calculated to evaluate the priority of the ten criteria. Members completed a matrix to indicate the relative priority or importance between items for achieving the goal (Cabala, 2010). The lesser or greater priority of each criteria against all of the others was assessed using a numerical nine-point scale.
The weights were then calculated from the pairwise comparisons to establish the priorities for all levels of the hierarchy: the ten criteria, subcriteria and key performance indicators. The geometric mean was used to calculate and determine the priority weights for the individual pairwise comparisons Then, the group priority weights were calculated by averaging the individual priority vectors using the weighted geometric mean (Dolan et al., 1989). All weights ranged in a scale between 0 and 1. Finally the results were evaluated for consistency using three key measures: consistency ratio, consistency index and the random index. To establish the consistency index $(\mathrm{Cl})$, the formula suggested by Saaty (1980) was used:

$$
C I=\frac{\lambda_{\max }-N}{n-1}
$$

Where ${ }^{\lambda} \max$ is the maximum eigenvalue of the matrix of the importance ratios and $n$ is the number of elements. The consistency ratio (CR) was calculated in order to determine if a pairwise comparison matrix was sufficiently consistent:

$$
C R=\frac{C I}{R I}
$$

This is calculated by using the ratio of the $\mathrm{Cl}$ to the random index (RI). The $\mathrm{RI}$ is a consistency index of a matrix of randomly generated comparisons.

\section{Results}

The main criteria of this research is composed of ten elements of crisis readiness: Risk and Hazard Management; Legal and Institutional Frameworks; Resources; Coordination; Information Management and Communication; Response Planning; Early Warning; Training; Recovery Initiation, and Property Protection. In addition to the main criteria, there are sub-criteria and key performance indicators for each criterion.

Analysis of the pairwise comparison for Crisis Readiness criteria shows that Response Planning was the highest ranked criterion with a relative weight of 0.173 , followed by Resources (0.154) and Training (0.147). These top three criteria accounted for nearly half $(47.4 \%)$ of the relative 
weights. As shown in Table 3 the next highest ranked criteria were Coordination (0.141), Information Management and Communication (0.114) and Risk and Hazard Management (0.096). Early Warning and Legal and Institutional Frameworks were moderately prioritised with weights of 0.056 and 0.055 respectively. The lowest ranked criteria were Recovery Initiation and Property Protection with respective weights of 0.038 and 0.026 . For each of the criteria the sub-criteria were evaluated to establish the relative importance of each item. It should be noted that due to practical limitations and time constraints the sub-criteria was only evaluated for the top six criteria of crisis readiness.

\section{Table 3 Prioritisation of Criteria}

\begin{tabular}{llc}
\hline \multicolumn{1}{c}{ Criteria } & Weight & Rank \\
\hline Response Planning & 0.173 & 1 \\
\hline Resources & 0.154 & 2 \\
\hline Training & 0.147 & 3 \\
\hline Coordination & 0.141 & 4 \\
\hline Information Management & 0.114 & 5 \\
and Communication & & 6 \\
\hline Risk and Hazard & 0.096 & 7 \\
\hline Management & & 8 \\
\hline Early Warning & 0.056 & 9 \\
\hline Legal and Institutional & 0.055 & 10 \\
\hline Framework & & \\
\hline Recovery Initiation & 0.038 & \\
\hline Property Protection & 0.026 & \\
\hline Consistency Measures & $\lambda_{\max }=10.88 ; C l=0.09 ; C R=0.06$ \\
\hline
\end{tabular}

For Response Planning as shown in Table 4 equal prioritisation was given to joint response planning and resource planning with weights of 0.365 . Roles and responsibilities ranked next with a weight of 0.172 while regular review and update was accorded the least priority of 0.099 . Of the six sub-criteria for Resources, human resources, physical resources and logistics and facilities capabilities ranked the highest and were all equally prioritised with weights of 0.244 . Weights for advanced technological resources, maintenance of logistics systems and logistics planning ranged between 0.106 and 0.065 . For the Training criterion continuous leadership development and field training ranked the highest with relative weights of 0.166 and 0.158 respectively. Exercises, technical training and equipment training were more moderately ranked with weights ranging between 0.123 and 0.113 . The least prioritised items were theoretical training, inter-agency training and adoption/benchmarking of best practice with relative weights ranging between 0.104 and 0.03 . Of the six sub-criteria for Coordination Operational Planning ranked the highest with a weight of 0.301 , followed by Coordinated Operational Procedures (0.259) and Inter-agency Coordination (0.177). Relative weights for the remaining sub-criteria of joint training, centralised coordination systems and public coordination ranged between 0.114 and 0.052 . For Information Management and Communication criterion, strategies and policies closely followed by advanced communication systems were the most prioritised with relative weights of 0.279 and 0.278 respectively. Public education and information programmes (0.133), multi-channel communications (0.110) and inter-agency communication (0.083) were ranked more moderately. For the lowest ranked sub-criteria of transfer of best practice, diverse communication forums and multiple incident reporting types priority weights ranged between 0.040 and 0.038 . For Risk and Hazard Management the highest ranked sub-criteria was Risk and Hazard Analysis with a significant weight of 0.417. Hazard management planning, documenting risks and forecasting and modelling ranked next with weights ranging between 0.203 and 0.118 . Hazard specific training had the least relative priority with a weight of 0.073 .

Furthermore, key performance indicators (KPIs) were evaluated for the six most highly ranked criteria. For Response Planning coordinated planning processes received the highest relative weight of 0.564 , followed by continuous planning (0.359) and qualified planners (0.077). Of the KPIs for Resources qualified human resource ratios ranked highest with a weight of 0.415 , one of the 
highest of any performance indicator. For the remaining KPIs of funding availability, technical resources availability, facilities and equipment availability and periodic review and maintenance relative weights ranged between 0.271 and 0.041 . For Training continuous training (0.198), training modes implemented (0.162), and frequency and types of exercises (0.160) were the most highly prioritised KPIs. Training evaluation and training and capacity standards were more moderately ranked with relative weights of 0.155 and 0.117 respectively. 
Table 4 Prioritisation of Sub-Criteria

\begin{tabular}{|c|c|c|c|c|}
\hline Criteria & Sub-Criteria & Weight & Rank & Consistency \\
\hline \multirow[t]{4}{*}{ Response Planning } & Joint Response Planning & 0.365 & 1 & \multirow{4}{*}{$\begin{array}{l}\lambda \max =4.19 \\
\mathrm{Cl}=0.06 \\
\mathrm{CR}=0.07\end{array}$} \\
\hline & Resource Planning & 0.365 & 2 & \\
\hline & Roles and Responsibilities & 0.172 & 3 & \\
\hline & Regular Review and Update & 0.99 & 4 & \\
\hline \multirow[t]{6}{*}{ Resources } & Human Resources & 0.244 & 1 & \multirow{6}{*}{$\begin{array}{l}\lambda \max =6.25 \\
C I=0.05 \\
C R=0.04\end{array}$} \\
\hline & Physical Resources & 0.244 & 2 & \\
\hline & Logistics and Facilities Capabilities & 0.244 & 3 & \\
\hline & Advanced Technological Resources & 0.106 & 4 & \\
\hline & Maintenance of Logistics Systems & 0.096 & 5 & \\
\hline & Logistics Planning & 0.065 & 6 & \\
\hline \multirow[t]{10}{*}{ Training } & Continuous Leadership Development & 0.166 & 1 & \multirow{10}{*}{$\begin{array}{l}\lambda \max =11.31 \\
\mathrm{Cl}=0.14 ; \\
\mathrm{CR}=0.09\end{array}$} \\
\hline & Field Training & 0.158 & 2 & \\
\hline & Exercises & 0.123 & 3 & \\
\hline & Technological Training & 0.116 & 4 & \\
\hline & Equipment Training & 0.113 & 5 & \\
\hline & Theoretical Training & 0.104 & 6 & \\
\hline & Inter-agency Exercises & 0.094 & 7 & \\
\hline & Virtual Simulations & 0.057 & 8 & \\
\hline & Database of Resources \& Capacities & 0.037 & 9 & \\
\hline & Benchmarking Best Practices & 0.030 & 10 & \\
\hline \multirow[t]{6}{*}{ Coordination } & Operational Planning & 0.301 & 1 & \multirow{6}{*}{$\begin{array}{l}\lambda \max =6.47 \\
\mathrm{Cl}=0.09 \\
\mathrm{CR}=0.07\end{array}$} \\
\hline & Coordinated Operational Procedures & 0.259 & 2 & \\
\hline & Interagency Coordination & 0.177 & 3 & \\
\hline & Joint Training & 0.114 & 4 & \\
\hline & Centralised Coordination Systems & 0.098 & 5 & \\
\hline & Public Coordination & 0.052 & 6 & \\
\hline \multirow[t]{5}{*}{ Risk \& Hazard Management } & Risk and Hazard Analysis & 0.417 & 1 & \multirow{5}{*}{$\begin{array}{l}\lambda \max =5.35 \\
\mathrm{Cl}=0.08 \\
\mathrm{CR}=0.08\end{array}$} \\
\hline & Hazard Management Planning & 0.203 & 2 & \\
\hline & Documenting Risks & 0.188 & 3 & \\
\hline & Forecasting and Modelling & 0.118 & 4 & \\
\hline & Hazard Specific Training & 0.073 & 5 & \\
\hline \multirow{8}{*}{$\begin{array}{l}\text { Information Management \& } \\
\text { Communication }\end{array}$} & Strategies and Policies & 0.279 & 1 & \multirow{8}{*}{$\begin{array}{l}\lambda \max =8.85 \\
\mathrm{Cl}=0.12 \\
\mathrm{CR}=0.08\end{array}$} \\
\hline & Advanced Communication Systems & 0.278 & 2 & \\
\hline & Public Education \& Information & 0.133 & 3 & \\
\hline & Multi-channel Communications & 0.011 & 4 & \\
\hline & Inter-agency Communication & 0.083 & 5 & \\
\hline & Transfer of Best Practice & 0.040 & 6 & \\
\hline & Diverse Communication Forums & 0.039 & 7 & \\
\hline & Multiple Incident Reporting Types & 0.038 & 8 & \\
\hline
\end{tabular}


Table 5 Rankings for Performance Indicators

\begin{tabular}{|c|c|c|c|c|}
\hline Criteria & Sub-Criteria & Weight & Rank & Consistency \\
\hline \multirow{3}{*}{$\begin{array}{l}\text { Response } \\
\text { Planning }\end{array}$} & Coordinated Planning Processes & 0.564 & 1 & \multirow{3}{*}{$\begin{array}{l}\lambda \max =3.07 ; \\
\mathrm{Cl}=0.03 ; \\
\mathrm{CR}=0.06\end{array}$} \\
\hline & Continuous Planning & 0.359 & 2 & \\
\hline & Qualified Planners & 0.077 & 3 & \\
\hline \multirow[t]{5}{*}{ Resources } & Qualified Human Resource Ratios & 0.415 & 1 & \multirow{5}{*}{$\begin{array}{l}\lambda \max =5.38 \\
\mathrm{Cl}=0.09 \\
\mathrm{CR}=0.08\end{array}$} \\
\hline & Funding Availability & 0.271 & 2 & \\
\hline & Technical Resources Availability & 0.137 & 3 & \\
\hline & Facilities and Equipment Availability & 0.137 & 4 & \\
\hline & Periodic Review and Maintenance & 0.041 & 5 & \\
\hline \multirow[t]{9}{*}{ Training } & Continuous Training & 0.198 & 1 & \multirow{9}{*}{$\begin{array}{l}\lambda \max =9.83 \\
C l=0.10 ; \\
C R=0.07\end{array}$} \\
\hline & Training Modes Implemented & 0.162 & 2 & \\
\hline & Frequency and types of exercises & 0.16 & 3 & \\
\hline & Training Evaluation & 0.155 & 4 & \\
\hline & Training and Capacity Standards & 0.117 & 5 & \\
\hline & Ratio of Accredited Training Programmes & 0.061 & 6 & \\
\hline & Number of Training Courses Implemented & 0.056 & 7 & \\
\hline & Multi-hazard Capacity Assessment & 0.054 & 8 & \\
\hline & Use of Virtual, Al and simulation technologies & 0.037 & 9 & \\
\hline \multirow[t]{6}{*}{ Coordination } & Equipped Operations Centres & 0.241 & 1 & \multirow{6}{*}{$\begin{array}{l}\lambda \max =6.47 ; \\
C l=0.09 ; \\
C R=0.07\end{array}$} \\
\hline & Crisis Appropriate Operational Protocols & 0.213 & 2 & \\
\hline & Information Exchange Mechanisms & 0.181 & 3 & \\
\hline & Inter-agency Coordination Mechanisms & 0.159 & 4 & \\
\hline & Defined Roles \& Responsibilities & 0.013 & 5 & \\
\hline & Public \& Community Coordination Mechanisms & 0.076 & 6 & \\
\hline \multirow{7}{*}{$\begin{array}{l}\text { Information } \\
\text { Management and } \\
\text { Communication }\end{array}$} & $\begin{array}{l}\text { Incident Report Communication time response } \\
\text { teams }\end{array}$ & 0.428 & 1 & \multirow{7}{*}{$\begin{array}{l}\lambda \max =7.78 ; \\
\mathrm{Cl}=0.13 ; \\
\mathrm{CR}=0.09\end{array}$} \\
\hline & Number of Information \& Reporting Mechanisms & 0.153 & 2 & \\
\hline & Smart Application Use in Incident Reporting & 0.121 & 3 & \\
\hline & Multilingual Communication Mechanisms & 0.121 & 4 & \\
\hline & Public Information Programmes Implemented & 0.083 & 5 & \\
\hline & Data Collection Mechanisms & 0.064 & 6 & \\
\hline & Trained Media Response Units & 0.031 & 7 & \\
\hline \multirow{5}{*}{$\begin{array}{l}\text { Risk and Hazard } \\
\text { Management }\end{array}$} & Continuous Hazard and Risk Monitoring & 0.288 & 1 & \multirow{5}{*}{$\begin{array}{l}\lambda \max =5.37 ; \\
C l=0.09 ; \\
C R=0.08\end{array}$} \\
\hline & All Hazards Risk Register & 0.260 & 2 & \\
\hline & Risk Communication Plan & 0.239 & 3 & \\
\hline & Plans for Specific Risks & 0.164 & 4 & \\
\hline & Frequency Risk Register Reviewed; & 0.049 & 5 & \\
\hline
\end{tabular}


As shown in Table 5 the least prioritised KPIs were ratio of accredited training programmes, number of training courses implemented, multi-hazard capacity assessment and use of virtual, $\mathrm{Al}$ and simulation technologies with relative weights ranging between 0.061 to 0.037 . Coordination equipped operations centres ranked highest (0.241), followed by crisis appropriate operational protocols (0.213) and information exchange mechanisms (0.181). Inter-agency coordination mechanisms, defined roles and responsibilities and public and community coordination mechanisms were next prioritised with weights ranging between 0.159 and 0.076 . For Information Management and Communication criterion communication time to response teams recorded a relative weight of 0.428 , the highest priority accorded to any performance indicator. Number of information and reporting mechanisms (0.153), smart application use in incident reporting (0.121), and multilingual communication mechanisms (0.121) ranked next in importance. Public information programmes implemented, data collection mechanisms and trained media response units were prioritised as least important with relative weights ranging between 0.083 to 0.031. For Risk and Hazard Management continuous hazard and risk monitoring ranked highest with a weight of 0.288 , while all hazards risk register, risk communication plan and frequency risk register reviewed relative weights ranged between 0.260 and 0.049 . The consensus responses conformed with acceptable requirements for the Consistency Ratio.

\section{Discussion}

The findings resulted in the ranking of the ten criteria that were identified for crisis readiness and response. Four criteria of Response Planning, Resources, Training, and Coordination were assigned the highest importance by practitioners. The AHP priority weights for these four criteria were in a narrow range suggesting these were viewed equal in significance. Thus the findings do not establish clearly the relative importance of these four criteria due to the marginal differences in the priorities assigned overall. Even so the priority attached to these four criteria by the
Delphi panel align with the key components of crisis readiness frameworks distinguished in the literature. These components consistently formed part of all of the frameworks reviewed in the literature review chapter (WHO, 2017; IASC, 2013; Sutton and Tierney, 2006; FEMA, 2001).

Response planning was the mostly highly ranked criterion overall but was only marginally prioritised over the other three top ranked criteria. While the majority of crisis readiness frameworks do not explicitly prioritise this criterion over others, all frameworks incorporate response planning as a core dimension (WHO, 2017; IASC, 2013; Sutton and Tierney, 2006; FEMA, 2001). One of the challenges acknowledged in the literature is the complexity in establishing flexible and responsive models. Evidence shows that overly prescriptive response plans can result in diminished ability to integrate situational factors and other contingencies during a crisis thus undermining response effectiveness (Berchtold et al., 2020; Quarantelli, 1998). The literature underlines the importance of autonomy and discretion for response planning and the impact of rigid structures. Evidence suggests a negative relationship between linear, command and control structures and response planning, and coordination failures as a result of the tendency to ignore the complexity and chaotic conditions inherent in crises (Ginter et al., 2006; Corbacioglu and Kapucu, 2005; Tierney, 2003). There is an implication towards development of open and autonomous cultures for countries such as the UAE where the national culture is characterised by high power distance and uncertainty avoidance (Hofstede, 2019).

Resources ranked as the second most important criterion for enhancing crisis response times. There is consistency in the literature and responses of the Delphi panel in the study of the relationship between resources and response planning. This finding supports the literature which consistently identifies the availability and management of resources as one of the most essential elements of crisis readiness and response (WHO, 2017; IASC, 2013; Sutton and Tierney, 2006; FEMA, 2001). However the 
literature emphasises extending beyond generic and simplified strategies to address the major resource constraints and competition that are typically experienced. This has implications for more advanced techniques for integration and optimisation of redundancy and resource-sharing based on more complex analyses. This is consistent with an interdependent and multifaceted dimension in crisis response associated with a range of different issues and factors (Wang and Sun, 2018; Hick et al., 2012; Simonoff et al., 2011). There are further implications for ensuring the efficient, fair and timely allocation of scarce resources that can account for specific crises and conditions (Choksi and Zaveri, 2019; Alsubaie et al., 2015). Evidence suggests that efficient resource allocation practice is increasingly evolving towards in-time and real-time techniques based on technology solutions such as RFID tagging, virtual simulation and resource management algorithms that prioritise and schedule resources (Choksi and Zaveri, 2019). An overarching challenge is the identification of antecedents including culture such as power that may influence cooperation inter-organisationally to promote optimisation and sharing of resources.

Panel members prioritised training as the third most important criterion for crisis readiness. This aligns with literature on the role of training as a key issue that directly impacts the ability of crisis response organisations to mount an effective response (Hošková-Mayerová, 2016; Bui and Subba, 2009). Training is cited in all of the crisis readiness frameworks reviewed for this study, although only the FEMA (2001) and IASC (2013) framework explicitly identify this criterion as a separate, discrete aspect. A key factor is the coordination of training between regions and agencies (Berchtold et al., 2017).

There is a major implication in defining key roles, structures and processes to account for diverse and specific training needs across an array of functions, roles and levels as well as intra and inter-organisational contexts and requirements (Adini et al., 2016; Leaning et al., 2013). While operational and tactical training is well established, gaps exist in training at strategic crisis management level and for novel or out-of-scale events (Owen et al., 2016). These issues have an implication for the assessment and evaluation of the effectiveness of training that can support the optimisation of training design across the entire crisis response community. Literature shows however that evaluation is frequently lacking and is in turn subject to numerous factors that can challenge or undermine the ability to assess training programmes for shortfalls and outcomes (Adini et al., 2016; Hsu et al., 2013).

Co-ordination was the fourth highest prioritised criterion and comparable to training. This finding lends support to the theoretical emphasis found in the literature as one of the principal elements directly connected to crisis readiness and crisis management during response and relief processes (Noori et al., 2016; Abbasi et al., 2013; Chen et al., 2008). While this criterion is reflected in the literature as a core element of crisis readiness, the extent to which it is addressed in different frameworks varies considerably (WHO, 2017; IASC, 2013; Sutton and Tierney, 2006; FEMA, 2001). The Common Framework for Preparedness (WHO, 2017) and the Strategic Framework for Emergency Preparedness (IASC, 2013) refer to coordination mechanisms among multiple actors across different levels and emphasise strategic vision but fall short of operationalising coordination mechanisms and procedures. The design of coordination mechanisms is dependent on overcoming differences in the organisational culture and therefore understanding of specific barriers and impediments in a particular context. A significant theme identified in the literature is the multiplicity and diversity of public administration and coordination structures which are influenced by different national and governance contexts, and often result in a range of hybrid and contradictory coordination arrangements (Christensen et al., 2016; Christensen et al., 2015). This suggests that countries such as the UAE need to develop the necessary conditions that can promote trust and cooperation. Arabic organisations are characterised by clandestineness, power dynamics, secrecy and closed, top-down cultures which may create challenges for development of 
coordination. To develop effective coordination for crisis readiness therefore has some implication for addressing cultural issues (Christensen et al., 2016).

Information Management and Communication (ICM) and Risk and Hazard Management received more moderate prioritisation in ratings and were the fifth and sixth highest criteria in terms of importance. The ICM dimension was identified as a key component of all the crisis readiness frameworks reviewed. The literature consistently shows consensus on the importance of rapid and accurate information gathering and dissemination to relevant actors and the public to mitigate the worst effects of crises and disasters (Sokat et al., 2016; OCHA, 2016).

Finally of all the criteria evaluated in the AHP four were perceived to be least important relative to the other criteria: Early Warning, Legal and Institutional Frameworks, Recovery Initiation and Property Protection. There was much less importance attached to these factors as evidenced by considerably lower ratings. Property Protection was perceived as least important of all ten criteria by a significant margin compared to the top criteria. While the relative significance of Recovery Initiation and Property Protection aligns with the literature, there is less consistency for Early Warning and Legal and Institutional Frameworks.

Examination of the existing frameworks indicates that these criteria are addressed to varying degrees with differing levels of detail. The Early Warning criteria is explicitly identified across all of the frameworks which specify necessary attributes and provide guidelines for effective early warning systems. Predominantly it is characterised as a key component of other criteria in the frameworks such as Resources (CP model) or Information Management (SFEP) (WHO, 2017; IASC, 2013; Sutton and Tierney, 2006; FEMA, 2001). Within the broader crisis readiness literature there is limited evidence of a specific research focus on early warning (Collins et al., 2008). Rather early warning has often been discussed within the context of overall hazard monitoring and forecasting (Hense et al., 2010; Singh and Subramaniam, 2009).
Legal and institutional frameworks are included in three of the four frameworks excluding the DP model and generally forms a distinct criterion. Discussion is detailed on the important components and dimensions for an appropriate legal and institutional context for enhancing crisis readiness, underlining the significance of developing policies and legislation that integrates emergency preparedness across sectors (WHO, 2017; IASC, 2013; FEMA, 2001).

Recovery Initiation is mentioned in two of the frameworks only and which diverge from each other in terms of the importance placed on this criterion. While the DP model (Sutton and Tierney, 2006) proposes recovery as a separate criterion and provides key elements and factors, in the SFEP framework Recovery Initiation is considered only as a component of response planning (WHO, 2017). The finding for Property Protection is reflected in the literature which overall places less emphasis on this criterion than most other criteria. Protecting property is represented in only one of the existing frameworks (DP model) that provides detail on the core components and elements (Sutton and Tierney, 2006).

A further goal of this study is to identify the key performance indicators (KPIs) for evaluating the effectiveness of different dimensions of CR. To achieve this aim practitioners generated a list of performance measures and process indicators in early rounds of Delphi that addressed different dimensions of crisis readiness. A shortlist of 35 KPIs for the top six criteria were then presented to participants in the AHP to evaluate and define their importance relative to each other. The results showed that for certain criteria priority was focused around a single or small number of indicators while for others the relative significance of each indicator was more balanced. While there is minimal definition of KPIs in the literature some indications lend weight to the findings of this study in underscoring the significance of the KPIs arising from this research. This identifies specific indicators classified under each of the top six criteria that support the development and evaluation of crisis readiness. For praxis these indicators represent a set of validated KPIs that 
can be integrated in existing crisis response plans to guide evaluation.

\section{Conclusion}

The increasing volatility of the environment arising either from human or natural events has placed greater significance in theory and praxis on crisis readiness and the capacity of institutions and society to respond effectively. The research problem for this study was stated within the context of the UAE and the gap between national and international practices in terms of response times of law enforcement and a comprehensive strategic framework that defines the critical success factors and performance indicators. While national level frameworks have been developed for major disasters and emergencies, sector or context-specific models have yet to be addressed.

The purpose of this study has been to investigate crisis readiness and response of Police in the UAE and establish the key dimensions that contribute to a comprehensive strategic framework that supports readiness and response planning to improve Police response times to road traffic crises. The qualitative and quantitative research conducted in this study provided primary data to identify and validate the key dimensions for crisis readiness. Findings from initial rounds of the Delphi process showed that of fourteen theoretical dimensions derived from theory and literature experts identified the top ten most critical components of crisis readiness. These criteria were then subject to an Analytical Hierarchy Process (AHP) in which the relative prioritisation and ranking of these criteria were identified. The results clustered into three distinct sets of ranking: four criteria of Response Planning, Resources, Training, and Coordination received the highest level of priority by the panel; Information Management and Communication and Risk and Hazard Assessment were ranked at mid-level importance. The remaining four criteria were accorded a lower level of priority: Early Warning, Legal and Institutional Frameworks, Recovery Initiation and Property Protection.

Qualitative findings further indicated a broad range of factors for each criterion proposed by practitioners. In a second phase of AHP experts were able to identify the relative importance of the sub-criteria for each of the top six criteria. These findings provide validation for the key criteria established in the literature and $C R$ frameworks and the relative priority of different factors and sub-criteria. Furthermore, qualitative data suggests a strong relationship and interdependency between different criteria and factors in this study. The findings from secondary and primary qualitative data suggested a number of antecedents associated with interorganisational, structural and national cultural factors as well as the application of different frameworks. Operational level antecedents were identified in respect of interoperability, resources, organisational culture and clandestineness and power. The results suggested that such factors impact on the efficiency and effectiveness of the existing approach to crisis readiness.

This research makes several contributions to theory and praxis in identifying the key dimensions and factors of crisis management and response. This research validates the key components of crisis readiness both theoretically based on a review of the literature and primary data validation by a panel of experts in the field based on a systematic analytical hierarchy process (AHP) approach. This has resulted in a novel framework that comprehensively addresses the key dimensions and factors of crisis readiness and provides a holistic model. Criteria and sub-criteria and key performance indicators for each criterion are ranked in order of priority and relative importance. This ranking can support the clarification of achievements and support identification of gaps and monitoring of factors and specific elements of crisis readiness. The framework supports practitioners as a management tool that supports strategic planning and decision-making for the development of organisational capacities that can enhance response times of police to road traffic crises. At the same they provide focus for further research into the impact of KPIs on the performance of crisis response agencies and institutions and programmes so that sub-elements can be identified and more closely aligned to critical 


\section{References}

Abbasi, A., Sadeghi-Niaraki, A., Jalili, M. and Choi, S.M., 2018. Enhancing response coordination through the assessment of response network structural dynamics. PloS one, 13(2), p.e0191130.

Acosta, J., Nelson C, Beckjord EB., 2009. A national agenda for public health systems research on emergency preparedness. [Online] Available at: <http://www.rand.

org/pubs/technical_reports/TR660.html>. [Accessed 22 ${ }^{\text {nd }}$ Nov 2021].

Adini, B., Ohana, A., Furman, E., Ringel, R., Golan, Y., Fleshler, E., Keren, U. and Reisner, S., 2016. Learning lessons in emergency management: the 4th International Conference on Healthcare System Preparedness and Response to Emergencies and Disasters. Disaster and military medicine, 2(1), p.16.

Al Marzooqi, A., 2017. Disaster risk reduction in the emirate of Abu Dhabi, United Arab Emirates: effects of natural and non-natural disasters over business continuity and sustainability. PhD Thesis: Université Sorbonne Paris Cité.

Alexander, D. 2012. Principles of emergency planning and management. $6^{\text {th }}$ ed. Edinburgh: Dunedin.

Alexander, D., 2009. Principles of emergency planning. In U. Fra Paleo (ed.) Building Safer Communities: Risk Governance, Spatial Planning and Responses to Natural Hazards. NATO Science for Peace and Security Series, Vol. 58. IOS Press, Amsterdam: 162-174.

Almarzouqi, I., 2017. An analysis of disaster vulnerability in the United Arab Emirates. PhD Thesis: Northumbria University.

Alshehri, S.A., Rezgui, Y. and Li, H., 2015. Disaster community resilience assessment method: a consensus-based Delphi and AHP approach. Natural Hazards, 78(1), pp.395-416.

Alsubaie, A., Alutaibi, K. and Marti, J., 2015. Resources allocation in emergency response using an interdependencies simulation environment. In: 2015 IEEE Canada International Humanitarian Technology Conference (IHTC2015), pp. 1-4. IEEE.

Alteneiji, H.R., 2015. A strategic approach to emergency preparedness in the UAE. PhD Thesis: University of Salford.

Berchtold, C., Vollmer, M., Sendrowski, P., Neisser, F., Müller, L. and Grigoleit, S., 2020. Barriers and Facilitators in Interorganizational Disaster Response: Identifying Examples Across Europe. International Journal of Disaster Risk Science, 11(1), pp.46-58.

Boin, A., Stern, E. and Sundelius, B., 2016. The politics of crisis management: Public leadership under pressure. Cambridge: Cambridge University Press. 
Borraz, O. and Cabane, L., 2017. States of crisis. Reconfiguring European States in Crisis, p.394.

Bui, T. and Subba, R., 2009. A tale of two disasters: Assessing crisis management readiness. In: 2009 42nd Hawaii International Conference on System Sciences, pp. 1-10. IEEE.

Cabała, P., 2010. Using the analytic hierarchy process in evaluating decision alternatives. Operations research and decisions, 20(1), pp.5-23.

CCA, 2004. Emergency preparedness: guidance on Part 1 of the Civil Contingencies Act 2004, its associated regulations and non-statutory arrangements. Easingwold: Emergency Planning College

Chen R, Sharman R, Rao H, Upadhyaya S., 2008. Coordination in emergency response management. Communications of the ACM., 51(5), pp.66-73.

Choksi, M. and Zaveri, M.A., 2019. Multiobjective Based Resource Allocation and Scheduling for Postdisaster Management Using IoT. In: Wireless Communications and Mobile Computing, 2019.

Christensen, T., Andreas Danielsen, O.L.E., Laegreid, P. E., 2016. Comparing coordination structures for crisis management in six countries. Public Administration, 94(2), pp.316332.

Christensen, T., Lægreid, P. and Rykkja, L.H., 2015. The challenges of coordination in national security management-the case of the terrorist attack in Norway. International Review of Administrative Sciences, 81(2), pp.352-372.

Collins, M., Neville, K., Hynes, W. and Madden, M., 2016. Communication in a disaster-the development of a crisis communication tool within the S-HELP project. Journal of Decision Systems, 25(sup1), pp.160-170.

Collins, M.L. and Kapucu, N., 2008. Early warning systems and disaster preparedness and response in local government. Disaster Prevention and Management: An International Journal, 17(5), pp.587-600.

Coppola, D.P., 2006. Introduction to international disaster management. London: Elsevier.

Corbacioglu, S. and Kapucu, N., 2005. Critical evaluation of Turkish disaster management: historical perspectives and new trends. The Turkish Public Administration Annual, 29(31), pp.53-72.

Cronin, J.K., 2015. Empowering Readiness: Influencing crisis management success outcomes. PhD thesis. Bond University, Australia.

Cutter, S.L., Burton, C.G. and Emrich, C.T., 2010. Disaster resilience indicators for benchmarking baseline conditions. Journal of Homeland Security and Emergency Management 7(1), Article 51.

Dillon, B., Dickinson, I., Whiteford, F., and Williamson, J., 2009. Emergency planning officers' handbook. Oxford: Oxford University Press.

Dranseika, V. and Gordijn, B. eds. 2018. Disasters: Core concepts and ethical theories. London: Springer International Publishing.

Drennan, L.T., McConnell, A. and Stark, A., 2014. Risk and crisis management in the public sector. London: Routledge.

EMAP, 2004. Building strong emergency preparedness communities. [online] Available at: <https://training.fema.gov/emiweb/downloads/d emers\%20\%20emap\%20em\%20accreditation\%20 program.pdf $>$ [Accessed 22 $2^{\text {nd }}$ Nov 2021].

Emergency Management Australia (EMA), 2004. Emergency management in Australia: Concepts and principles. [online] Available at: <https://knowledge.aidr.org.au/media/1972/ma nual-1-concepts-and-principles.pdf> [Accessed $22^{\text {nd }}$ Nov 2021].

Eriksson, K., 2015. Framework for crisis preparedness planning: Four required areas for developing a learning process. Journal of Emergency Management (Weston, Mass.), 13(6), pp.519-531.

EU, 2018. European emergency response capacity. [online] Available at: <https://ec.europa.eu/echo/what/civilprotection/european-emergency-responsecapacity_en $\geq$ [Accessed $22^{\text {nd }}$ Nov 2021].

Feldmann-Jensen, S., Jensen, S. and Smith, S.M., 2017. The next generation core competencies for emergency management professionals: Handbook of behavioral anchors and key actions for measurement. FEMA. [online] Available at: $<$ https://training.fema.gov/hiedu/docs/emcompe tencies/final_\%20ngcc_and_measures_aug2017. pdf $>$ [Accessed 22 ${ }^{\text {nd }}$ Nov 2021]. 
FEMA, 2001. State capability assessment for readiness. [Online] Available at: <https://usfa.kohalibrary.com/app/work/167674 $>$ [Accessed 22 ${ }^{\text {nd }}$ Nov 2021].

FEMA, 2015. National Preparedness; goals and core capabilities. [Online] Available at: <https://www.fema.gov/national-preparedness> [Accessed 22 ${ }^{\text {nd }}$ Nov 2021].

Ginter, P.M., Duncan, W.J., McCormick, L.C., Rucks, A.C., Wingate, M.S. and Abdolrasulnia, M., 2006. Effective response to large-scale disasters: the need for high-reliability preparedness networks. International Journal of Mass Emergencies and Disasters, 24(3), p.331.

Gordon, J., 2002. Comprehensive emergency management for local governments: demystifying emergency planning. Brookfield, Conn.: Rothstein Associates.

Hense, K.A., Wyler, B.D. and Kaufmann, G., 2010. Preparedness versus reactiveness: an approach to pre-crisis disaster planning. Journal of Homeland Security and Emergency Management, 7(1).

Henstra, D., 2010. Evaluating local government emergency management programs: What framework should public managers adopt?. Public Administration Review, 70(2), pp.236-246.

Hick, J.L., Hanfling, D. and Cantrill, S.V., 2012. Allocating scarce resources in disasters: emergency department principles. Annals of emergency medicine, 59(3), pp.177-187.

Hoffer Gittel, J., Cameron, K., Lim, S. and Rivas, V., 2006. Relationships, layoffs, and organizational resilience: Airline industry responses to September 11. The Journal of Applied Behavioral Science, 42(3), pp.300-329.

Hofstede, 2019. United Arab Emirates. [Online] Available at: <https://www.hofstedeinsights.com/country/the-united-arab-emirates/> [Accessed 22 ${ }^{\text {nd }}$ Nov 2021].

Hošková-Mayerová, Š., 2016. Education and Training in Crisis Management. ERIC.

Hsu, E.B., Li, Y., Bayram, J.D., Levinson, D., Yang, S. and Monahan, C., 2013. State of virtual reality based disaster preparedness and response training. PLoS currents, 5.

Ikeda, S., 2010. An interdisciplinary approach to disaster risk communication from the long-term perspective of risk governance. In: International Disaster and Risk Conference IDRC Davos 2010, Proceedings, Davos, Switzerland 2010.

Inter-Agency Standing Committee (IASC), 2013. Common Framework for Preparedness. [Online] Available at: <https://interagencystandingcommittee.org/syst em/files/iasc_common_framework_for_prepared ness.pdf> [Accessed 22 ${ }^{\text {nd }}$ Nov 2021].

Kartez, J. D, and Lindell, M. K., 1987. Planning for uncertainty: The case of local disaster planning. J Am Plann Assoc. 53(4), pp. 487- 498.

Kovoor-Misra, S., 1995. A multidimensional approach to crisis preparation for technical organizations: Some critical factors. Technological Forecasting and Social Change, 48, pp. 143-160.

Lampel, J., Shamsie, J., and Shapira, Z., 2009. Experiencing the improbable: Rare events and organizational learning. Organization Science, 20(5), pp. 835-845.

Leaning, J. and Guha-Sapir, D., 2013. Natural disasters, armed conflict, and public health. New England journal of medicine, 369(19), pp.18361842.

Lettieri, E., Masella, C. and Radaelli, G., 2009. Disaster management: findings from a systematic review. Disaster Prevention and Management: An International Journal.

Light, P.C. and Morgan, S., 2008. Predicting organizational crisis readiness: Perspectives and practices toward a pathway to preparedness. New York University: Center for Catastrophe Preparedness and Response.

Ministry of Interior, 2017. National project time reduction indicator: Responding to all emergency communications in minutes. Internal Publication.

Nagasaka, T., 2006. E-community platform of disaster risk communication enhanced by information technologies recent pilot studies in local communities in Japan. In: International Disaster and Risk Conference IDRC Davos 2006, Proceedings, Davos, Switzerland 2006.

National Research Council (NRC), 2006. Facing Hazards and Disasters: Understanding Human Dimensions. The National Academies Press: Washington, D.C. 
Noori, N.S., 2016. Coordination dynamics in disaster response operations: A network based discrete event analysis. PhD Thesis. Universitat Ramon LLull.

OECD, 2013. Strategic crisis management. [online] Available at: <https://www.mmc.com/content/dam/mmcweb/Files/Strategic-Crisis-Management-paperJuly-2013.pdf> [Accessed $22^{\text {nd }}$ Nov 2021].

Owen, C., Bosomworth, K., Bearman, C., Brooks, B., Fogarty, L. and Conway, G., 2016. Politics, policies and paradigms, challenges of change in future emergency management. Bushfire CRCAFAC, 13.

Perry, M., 2007. Natural disaster management planning. International Journal of Physical Distribution \& Logistics Management.

Quarantelli, E. L., 1998. Major criteria for judging disaster planning and managing and their applicability in developing societies. Newark, Delaware: Disaster Research Center, University of Delaware, p. 268.

Reilly, A.H., 1987. Are organizations ready for crisis-a managerial scorecard. Columbia Journal of world business, 22(1), pp.79-88.

Richey, J.M. and Grinnell, M., 2004. Evolution of roadmapping at Motorola. Research Technology Management, 47(2), pp. 37-40.

Ritchie, B. W., Bentley, G., Koruth, T. and Wang, J., 2011. Proactive crisis planning: Lessons for the accommodation industry. Scandinavian Journal of Hospitality and Tourism, 11(3), pp. 367-386.

Rousaki, B. and Alcott, P., 2006. Exploring the crisis readiness perceptions of hotel managers in the UK. Tourism \& Hospitality Research, 7(1), pp.2738.

Saaty TL., 1980. The analytical hierarchy process, planning, priority. resource allocation. New York: RWS Publications

Saaty, T.L., 1978. Modeling unstructured decision problems-the theory of analytical hierarchies. Mathematics and computers in simulation, 20(3), pp.147-158.

Sheaffer, Z. and Mano-Negrin, R., 2003. Executives' orientations as indicators of crisis management policies and practices. Journal of Management Studies, 40(2), pp. 573-606.
Shrivastava, P. and Mitroff, I.I., 1987. Strategic management of corporate crises. Columbia World Journal of Business, 22, no. 1, p. 7.

Simonoff, J.S., Restrepo, C.E., Zimmerman, R., Naphtali, Z.S. and Willis, H.H., 2011. Resource allocation, emergency response capability, and infrastructure concentration around vulnerable sites. Journal of Risk Research, 14(5), pp.597-613.

Singh, H. and Subramaniam, S., 2009. Health emergency and disaster preparedness in Malaysia. Southeast Asian Journal of Tropical Medicine and Public Health, 40, p.11.

Sokat, K.Y., Zhou, R., Dolinskaya, I.S., Smilowitz, K. and Chan, J., 2016. Capturing real-time data in disaster response logistics. Journal of Operations and Supply Chain Management, 9(1), pp.23-54.

Sutton, J. and Tierney, K., 2006. Disaster preparedness: Concepts, guidance, and research. Colorado: University of Colorado.

Tierney, K., 2003. Disaster beliefs and institutional interests: Recycling disaster myths in the aftermath of 9-11. In :Terrorism and disaster: New threats, new ideas, pp. 33-51. New York: Emerald Group Publishing Limited.

Van Wart, M. and Kapucu, N., 2011. Crisis management competencies: The case of emergency managers in the USA. Public Management Review, 13(4), pp.489-511.

Wang, Y. and Sun, B., 2018. A multiobjective allocation model for emergency resources that balance efficiency and fairness. In: Mathematical Problems in Engineering, 2018.

WHO, 1999. Disaster management: Institutional framework. [online] Available at: <http://apps.who.int/disasters/repo/5508.pdf $\geq$ [Accessed 22 ${ }^{\text {nd }}$ Nov 2021].

WHO, 2017. A strategic framework for emergency preparedness. [Online] Available at: <http://www.who.int/ihr/publications/97892415 11827/en/ $\geq$ [Accessed $22^{\text {nd }}$ Nov 2021].

WHO, 2020. Global status report on road safety 2018. [Online] Available at: $<$ https://www.who.int/publications-detail/globalstatus-report-on-road-safety-2018> [Accessed $22^{\text {nd }}$ Nov 2021].

Willyard, C.H. and McClees, C.W., 1987. 
Motorola's technology roadmap process. Research Management (Sep-Oct), pp.13-19.

WTTC, 2019. Crisis Readiness: Are You Prepared And Resilient To Safeguard Your People \& Destinations? World Travel \& Tourism Council.
Zemp, H., 2010. ICT and effective disaster management in a changing media world The critical function of disaster communications in dynamic and diverse contexts by its users In: International Disaster and Risk Conference IDRC Davos 2010, Proceedings, Davos, Switzerland 2010. 